Supporting Information

\title{
Hyperspectral luminescence imaging in combination with signal deconvolution enables reliable multi-indicator based chemical sensing
}

\author{
Silvia E. Zieger*1, Maria Moßhammer², Michael Kühl² and Klaus Koren*1 \\ ${ }^{1}$ Aarhus University Centre for Water Technology (WATEC), Department of Biology, Section for Microbiology, Aarhus University, \\ 8000 Aarhus C, Denmark \\ ${ }^{2}$ Marine Biological Section, Department of Biology, University of Copenhagen, 3000 Helsingør, Denmark \\ *Corresponding Author: szieger@bio.au.dk and klaus.koren@bio.au.dk
}

\section{CONTENT}

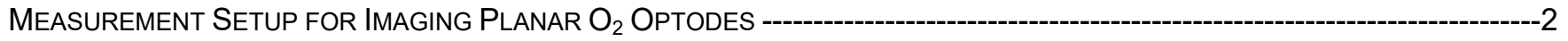

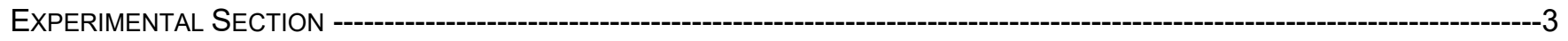

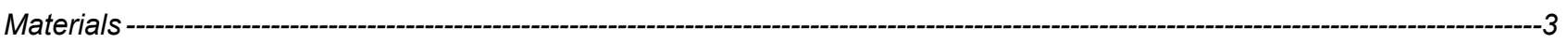

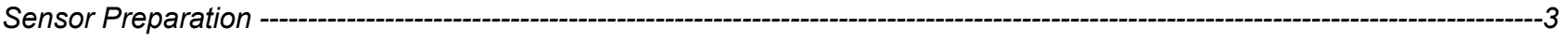

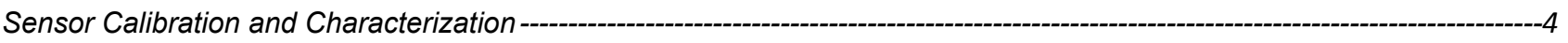

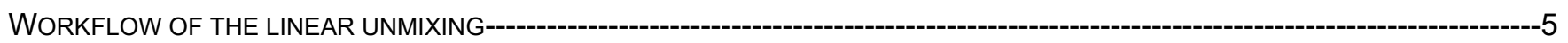

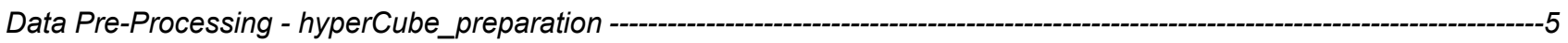

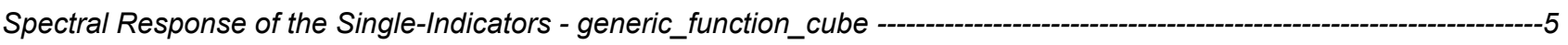

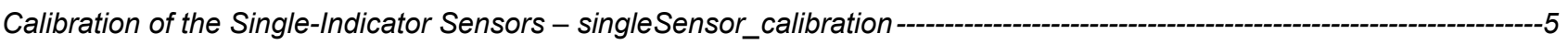

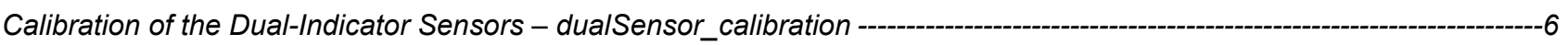

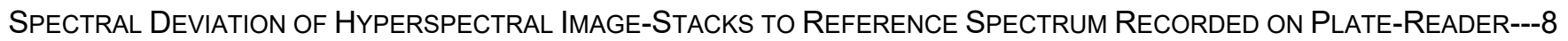

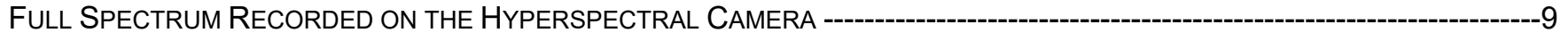

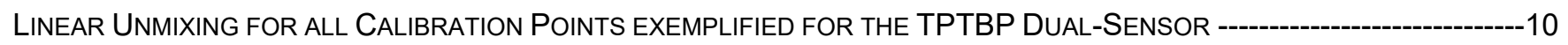

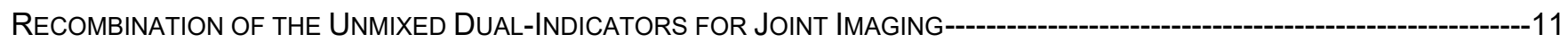

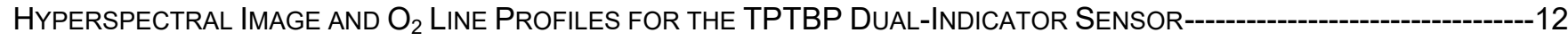

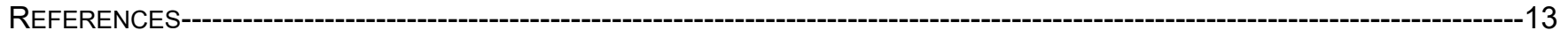




\section{Measurement Setup for Imaging Planar $\mathrm{O}_{2}$ Optodes}

(A)

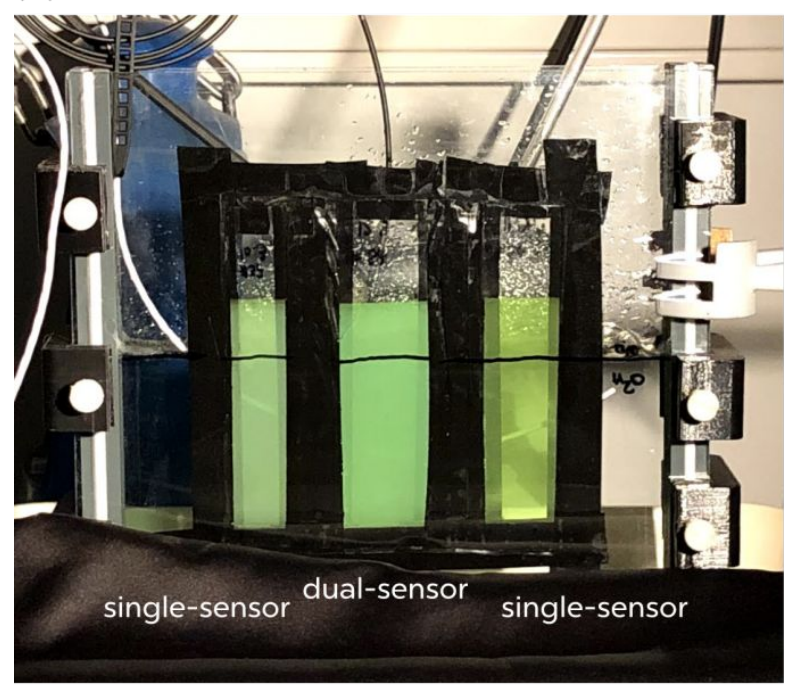

(B)

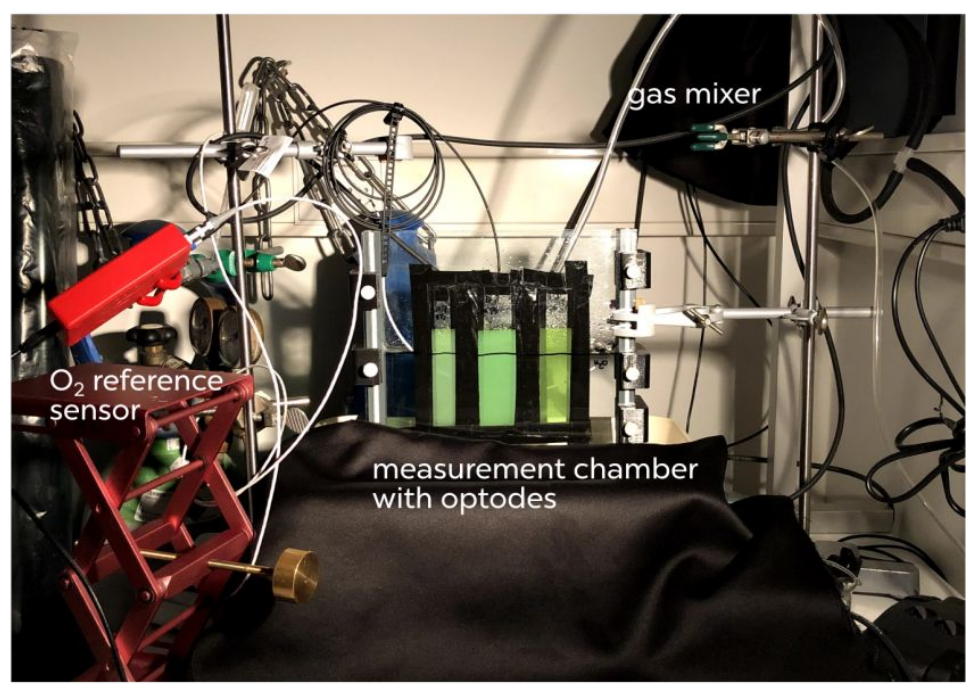

(C)

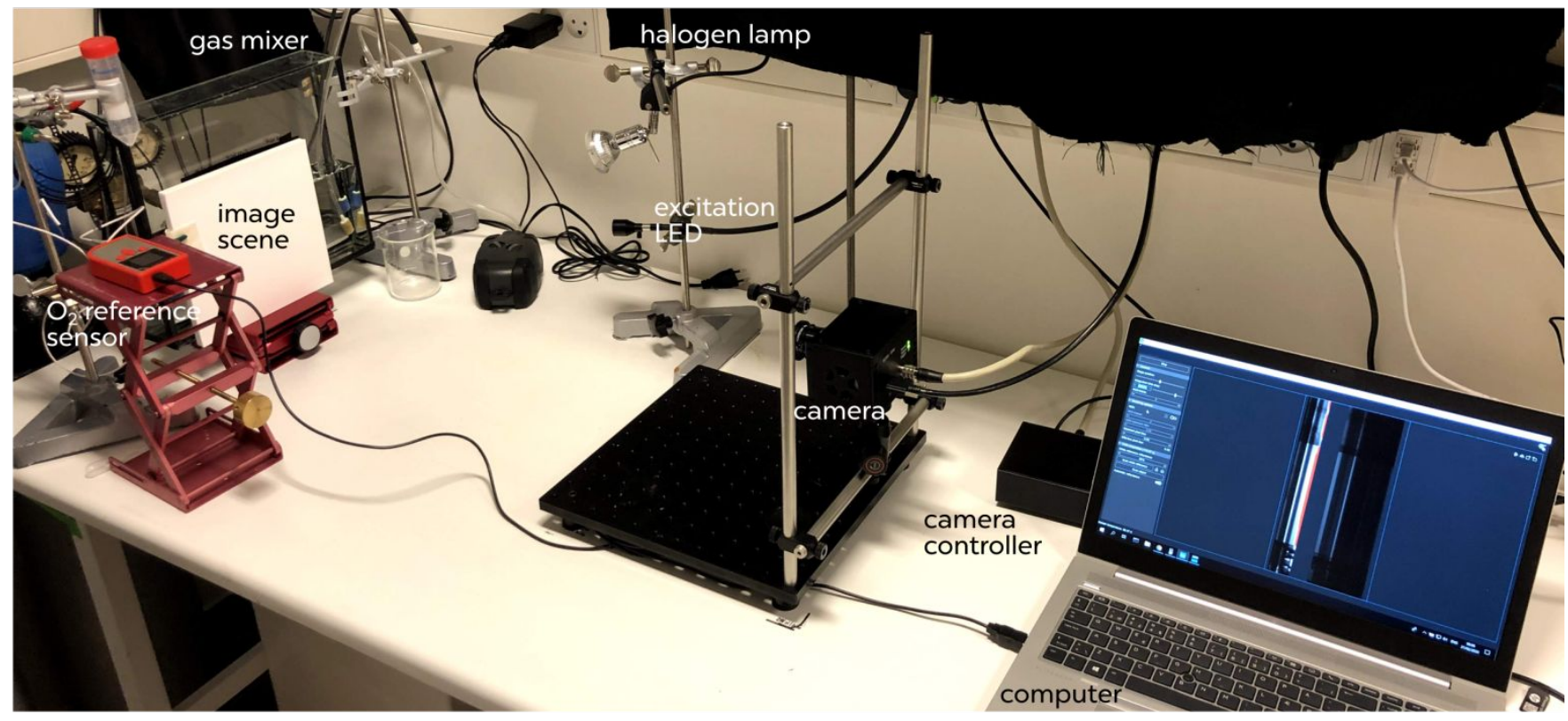

Figure $\mathrm{S} 1$ Measurement chamber and measurement setup used to calibrate single- and dual-sensors for $\mathrm{O}_{2}$. (A) The respective sensor foils (dual-sensor in the middle framed by the single-sensor foils on the sides) were taped on the inside of the glass wall, mounted inside the calibration chamber. (B) The calibration was done in the water phase by altering the $\mathrm{O}_{2}$ concentration with the help of a PC-controlled gas mixer. The fiber-optic reference sensor monitored temperature and $\mathrm{O}_{2}$ concentration. (C) The PC-controlled hyperspectral camera was positioned in front of the calibration chamber with the excitation light mounted at an angle to avoid reflectance and optical cross talk with the camera. The halogen lamp and the reflectance tile were used for camera calibration. 


\section{Experimental Section}

Materials

The $\mathrm{O}_{2}$-sensitive red-emitting porphyrin indicators (platinum(II)-5,10,15,20-tetrakis-(2,3,4,5,6-pentafluorphenyl)porphyrin (Pt-TFPP; $\lambda_{\mathrm{em}, \max }=652 \mathrm{~nm}$ when immobilized in the sensor foil) and palladium(II)-5,10,15,20-tetrakis(2,3,4,5,6-pentafluorphenyl)-porphyrin (Pd-TFPP; $\left.\lambda_{\mathrm{em}, \max }=674 \mathrm{~nm}\right)$ ), and the NIR-emitting benzoporphyrin indicators (platinum(II)-meso-tetraphenyl-tetrabenzoporphyrin (Pt-TPTBP; $\lambda_{\mathrm{em}, \max }=779 \mathrm{~nm}$ ) and palladium(II)meso-tetraphenyl-tetrabenzoporphyrin (Pd-TPTBP; $\lambda_{\mathrm{em}, \max }=773 \mathrm{~nm}$ )) were obtained from Frontier Scientific (frontiersci.com , Logan, US). The reference dye Macrolex Fluorescence Yellow (MFY 10GN) was bought from Lanxess AG (lanxess.com, Köln, Germany), and monocrystalline diamond powder was purchased from Microdiamant AG (microdiamant.com, Lengwil, Switzerland). Polystyrene (PS, MW 250 00o), sodium sulfite $\left(\mathrm{Na}_{2} \mathrm{SO}_{3}\right)$, chloroform and toluene were obtained from Sigma Aldrich (sigmaaldrich.com, St Louis, U.S.). The PET

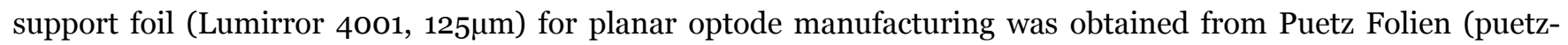
folien.com, Taunusstein, Germany). All chemicals were used as received.

\section{Sensor Preparation}

\section{Single and dual-indicator porphyrin (TFPP) sensor}

For single indicator sensor foils, only one of the TFPP analog dyes was used in combination with the MFY reference. For the Pd-sensor, $2.28 \mathrm{mg}$ Pd(II)-porphyrin indicator were dissolved in $1.5 \mathrm{~g}$ 10\% w/w PS (in chloroform), whereas

2.25 mg Pt(II)-porphyrin were used for the Pt-analog. In each case, $2.27 \mathrm{mg} \mathrm{MFY}$ and $150 \mathrm{mg}$ diamond powder were added as reference and as signal enhancer, respectively. For the dual indicator $\mathrm{O}_{2}$-sensitive sensor foil, a sensor cocktail was prepared incorporating both porphyrin analogs and the reference dye: $2.24 \mathrm{mg}$ of Pt(II)-porphyrin and $2.04 \mathrm{mg}$ of Pd(II)-porphyrin were dissolved in $1.5 \mathrm{~g}$ 10\% w/w PS (in chloroform). MFY (2.22 mg) and diamond powder $(150 \mathrm{mg}$ ) were added to the sensor cocktail as reference and signal enhancer, respectively.

Single and dual-indicator benzoporphyrin (TPTBP) sensor

The same procedure as for the porphyrin dyes was conducted for the single- and dual-indicator sensors based on the benzoporphyrin analogs. 2.27 mg were used for either Pd(II)-benzoporphyrin or Pt(II)-benzoporphyrin. The respective indicator dye was dissolved in $1.5 \mathrm{~g}$ 10\% w/w PS (in toluene), $3.8 \mathrm{mg}$ of MFY were added as reference, and $150 \mathrm{mg}$ diamond powder were added as signal enhancer. For the dual $\mathrm{O}_{2}$-indicator sensor foil, a sensor cocktail was prepared using both benzoporphyrin analogs and the reference dye. $1.84 \mathrm{mg}$ of Pt-TPTBP and $2.32 \mathrm{mg}$ of PdTPTBP were dissolved in $1.5 \mathrm{~g}$ 10\% w/w PS (in toluene). MFY (4.37 mg) and diamond powder (150 mg) were added to the sensor cocktail as reference and signal enhancer, respectively.

For planar optode fabrication, the sensor cocktails were knife coated onto dust free PET films using a film applicator (Byk-Gardner GmbH, Germany) yielding a $\sim 12 \mu \mathrm{m}$-thick sensor layer on top of the transparent carrier foil after solvent evaporation. 


\section{Sensor Calibration and Characterization}

The dual-indicator foil (approx. $2.5 \times 7 \mathrm{~cm}^{2}$ ) was taped on the inner transparent glass wall of a water-filled measurement chamber, framed by the respective single-indicator foils of approximately the same dimensions. Excitation and imaging of sensor foils was done through the chamber wall (Fig. S 1). For calibration, the dissolved oxygen levels in the water were altered between 950 to o hPa using compressed $\mathrm{N}_{2}$ and $\mathrm{O}_{2}$ bought from Air Liquide S.A. (airliquide.dk; Taastrup, Denmark) in combination with a gas mixer (Red-y-compact; Vögtlin Instruments $\mathrm{GmbH}$; Muttenz, Switzerland). At the lowest $\mathrm{O}_{2}$ level, sodium sulfite was added as an extra $\mathrm{O}_{2}$ scavenger to ensure completely anoxic conditions. The calibrations and the subsequent experiments were performed at the same constant temperature $\left(22.5 \pm 0.5{ }^{\circ} \mathrm{C}\right)$. A luminescent based fiber-optic phase-fluorimeter (FireSting GO2; PyroScience $\mathrm{GmbH}$, Aachen, Germany) equipped with a robust $\mathrm{O}_{2}$ sensor (OXROB3; PyroScience GmbH, Aachen, Germany) was used as a reference $\mathrm{O}_{2}$ sensor to monitor the temperature and the dissolved oxygen concentrations in the calibration chamber. The reference sensor was calibrated according to the manufacturer's instructions using a two-point calibration with anoxic and air saturated water.

The spectral characterization of the $\mathrm{O}_{2}$ optodes was done using a microplate reader (Clariostar Plus; bmglabtech.com; Birkerød, Denmark). A disc with a diameter of approximately $22 \mathrm{~mm}$ was cut out from the sensor foil and positioned in a microplate well. The well was filled with anoxic water $\left(2 \% \mathrm{w} / \mathrm{w}\right.$ of $\left.\mathrm{Na}_{2} \mathrm{SO}_{3}\right)$ to characterize the sensor foils at its maximal luminescence intensity (Fig. S 2). The luminescence spectra of the TPTBP single- and dual-indicator sensors were recorded between $460-840 \mathrm{~nm}$ with a slit width of $10 \mathrm{~nm}$ and a wavelength step of $1 \mathrm{~nm}$. The excitation wavelength was set according to the maximal intensity of the reference dye (440 nm; $10 \mathrm{~nm}$ slit). For the excitation spectra, the sensor foils were scanned between $350-700 \mathrm{~nm}$ at a slit width of $10 \mathrm{~nm}$ and a step width of $1 \mathrm{~nm}$. The emission wavelength was set to the maximal luminescence of the respective indicator (PtTPTBP $770 \mathrm{~nm}$; Pd-TPTBP $796 \mathrm{~nm}$; dual-indicator sensor $770 \mathrm{~nm}$ ). To characterize the spectral properties of the TFPP sensors, their luminescence spectra were recorded between 420-750 $\mathrm{nm}$ with an excitation wavelength of $400 \mathrm{~nm}$. The excitation spectra were recorded between $320-640 \mathrm{~nm}$ and the emission wavelength was set to $650 \mathrm{~nm}$ (Pt-TFPP and dual-indicator sensor) or $670 \mathrm{~nm}$ (Pd-TFPP), respectively. In each case, the slit width was $10 \mathrm{~nm}$ and the step width $1 \mathrm{~nm}$.

Limit of detection (LoD) of the single and dual-indicator sensors

According to Shrivastava ${ }^{1}$, the detection limit of both, the single indicator and the dual-indicator dyes can be determined according to equation (eq. S 1):

$$
y=x(\text { blank })+3 \sigma(\text { blank })
$$

where $x$ is the average signal intensity of the indicator and $\sigma$ is the respective standard deviation when the blank, e.g. the anoxic calibration point, is measured. To ultimately determine the lowest measurable $\mathrm{O}_{2}$ concentration, we insert the results from equation (eq. S 1) in a transformed simplified Stern-Volmer equation (cf eq. S 2). 


\section{Workflow of the linear unmixing}

Acquired images (hyperspectral data cubes) were radiometrically corrected using the angularity model described by Pichette et al. ${ }^{2}$. The correction is done against a white reference tile under known light conditions covering the whole wavelength range of the camera. The correction is done using a MATLAB script that can be obtained from the camera manufacturer upon request.

The following procedures were programmed in Python 3.7.4. All required libraries were up to date at the time the paper was submitted. The programming code can be downloaded from GitHub (github.com/silviaelisabeth/Signaldeconvolution-in-optical-chemical-imaging-for-reliable-multi-analyte-sensing) and is openly accessible.

\section{Data Pre-Processing - hyperCube_preparation}

All acquired and radiometrically corrected hyperspectral cubes are pre-processed in order to remove redundant information to accelerate subsequent evaluation steps.

The cubes are loaded using the Spectral Python (SPy) package dedicated to process hyperspectral image data. For ease of use, the hyperspectral cube is transformed so that individual image frames can be accessed via the wavelength. If the user selects regions of interest (RoIs), the algorithm extracts the spectral information within those regions, calculates the average intensity along the wavelength range within each RoI, and stores the full 3Dmatrix as well as the average spectrum in a dictionary for further analysis.

\section{Spectral Response of the Single-Indicators - generic_function_cube}

The pre-processed hyperspectral image-stacks, or rather the RoIs, for the three lowest $\mathrm{O}_{2}$ concentration points are used to determine the generic function for the single-indicators. The RoIs are extracted as described before including the averaging of the spectra. The average spectra are then baseline corrected and normalized to the maximal indicator intensity. Subsequently, the whole spectrum is split into parts to select the region, where the reference or the indicator emits and where background noise is interfering.

The generic mathematical description for the spectral response of the single indicators is then determined by curve fitting of the sample data using the Non-Linear Least-Squares Fitting (lmfit) Python package and a customdesigned model. The model assumes that the spectra of the reference and the indicator dyes are best described by a Voigt profile, while any background noise can be fitted by a Gaussian profile ${ }^{3}$. The generic functions for each indicator and the corresponding fit parameters are stored in a txt-file and an hdf5-file, respectively.

For validation, the spectral deviation of the determined generic function can be compared to the corresponding reference spectra, which are recorded on a state-of-the-art spectrometer. The spectral deviation of all singleindicators from their corresponding reference spectra is shown in Figure S 2.

Calibration of the Single-Indicator Sensors - singleSensor_calibration

Calibration files are loaded and preprocessed, if required. The reference sensor provides the exact analyte concentration of each calibration point. Subsequently, the algorithm removes outliers and merges replication of the same concentration point by averaging the respective spectra. For the actual calibration method, an integral approach is chosen, where the user defines the wavelength ranges for the reference and the indicator dyes and the 
spectra are cropped accordingly. Table S 1 lists the integration limits for the indicator and the reference of each optode. Within these ranges, the integral of both dyes is determined using Simpson's rule (also known as Kepler's rule), i.e., a numerical approximation of definite integrals ${ }^{4}$. The ratio of the integrals of both dyes is then formed (mean and standard deviation) at each calibration point.

The ratio is then fitted against the analyte concentration using an exponential decay fit following the simplified Stern-Volmer Fit as it is commonly done for $\mathrm{O}_{2}$ sensors ${ }^{5,6}$.The fit is executed using the lmfit package with a customdesigned model following the equation for a modified Stern-Volmer Fit using the two-site model 7,8:

$$
\frac{I}{I_{0}}=\frac{f}{1+K_{s v} \cdot x}+(1-f)
$$

eq. S 2

where $x$ is the oxygen concentration, $I$ and $I_{o}$ are the intensity at a certain oxygen concentration and at o hPa, respectively. $K_{s v}$ is the Stern-Volmer quenching constant and $f$ and $(1-f)$ are the quenchable and non-quenchable fraction of the immobilized indicator.

Table S 1 Integration limits for each single indicator used to determine the integral according to Simpson's rule for numerical integration of definite integral

\begin{tabular}{lcc} 
Sensor & $\begin{array}{r}\text { Integration limits reference } \\
(\mathrm{nm})\end{array}$ & $\begin{array}{c}\text { Integration limits indicator } \\
(\mathrm{nm})\end{array}$ \\
Pd-TFPP & $490-580$ & $600-800$ \\
Pt-TFPP & $490-580$ & $600-800$ \\
\hline Pd-TPTBP & $490-580$ & $660-900$ \\
Pt-TPTBP & $490-580$ & $660-900$
\end{tabular}

Calibration of the Dual-Indicator Sensors - dualSensor_calibration

The generic function of the single-indicators and the calibration files of the superimposed signals are loaded. When using the radiometrically corrected measurement files, the pre-processing function must be applied to the hyperspectral cubes. The reference sensor provides the exact analyte concentration of each calibration point. Outliers are removed and replications of the same concentration point are averaged.

Before the actual signal deconvolution, the superimposed signal and the generic functions of the single-indicators are aligned, ensuring that both exhibit the same wavelength range. If required, the generic function is interpolated to the same wavelength range as the superimposed signal.

Afterwards, the actual function for linear unmixing is applied. The assumption is that the measured signal representing the superimposed signal of two indicators can be described as the sum of two single indicators multiplied with a respective coefficient that changes with the $\mathrm{O}_{2}$ concentration. The coefficients only depends on the $\mathrm{O}_{2}$ concentrations assuming that no interaction between the indicators takes place, even though they spectrally overlap. 


$$
\psi\left(\lambda, O_{2}\right)=\alpha\left(O_{2}\right) \cdot \varphi(\lambda, P d)+\beta\left(O_{2}\right) \cdot \varphi(\lambda, P t)
$$

eq. S 3

where $\varphi$ is the generic function of the single-indicator and $\alpha$ and $\beta$ are the $\mathrm{O}_{2}$-sensitive coefficients. $\psi$ is the superimposed signal measured with the hyperspectral camera.

Equation (eq. S 3) can easily be transferred into an optimization problem (eq. S 4), where the objective function must be optimized according to:

$$
\chi^{2}=\sum_{\lambda=i}^{n} \frac{(\Phi(\lambda)-\psi(\lambda))^{2}}{n-2}
$$

eq. S 4

where $\Phi$ is the theoretical sum defined by equation (eq. S 3), $\psi$ is the measured, superimposed signal and $n$ is the length of the sample data. The parameter of choice to be minimized is the $\chi$ value, describing the goodness of fit of how well the theoretical sum of the single-indicators multiplied with the respective coefficients coincides with the measured, superimposed signal 9. The optimization problem is addressed using the minimize function of SciPy (scipy.optimize) returning the optimal $\chi$ value and the corresponding coefficients $(\alpha, \beta)$ for the single indicators. For solving, we found that the SLSQP (sequential least-squares programming) method worked best resulting in a minimal $\chi^{2}$ value.

The integration of the coefficients multiplied with the generic function and ratio-determination is done as described for the single-indicators. The integral ratios are fitted against the analyte concentration assuming a modified SternVolmer Fit incorporating the two-site model 7,8 . The results of the Stern-Volmer Fit and the calculated LoD are listed in Table $\mathrm{S} 2$.

\begin{tabular}{|c|c|c|c|c|c|c|}
\hline & & $f[1]$ & $K_{s v}\left[\mathrm{hPa}^{-1}\right]$ & $K_{s v}$ ratio $(P d / P t)$ & $x^{2}$ & $\operatorname{LoD}[\mathrm{hPa}]$ \\
\hline \multirow[t]{4}{*}{ Single indicator } & Pd-TFPP & 0.949 & 0.107 & 6.69 & $1.50 \mathrm{e}-1$ & 0.15 \\
\hline & Pt-TFPP & 0.938 & 0.016 & & $3.02 \mathrm{e}-3$ & 2.87 \\
\hline & Pd-TPTBP & 0.988 & 0.054 & 3.18 & $5.69 \mathrm{e}-1$ & 0.73 \\
\hline & Pt-TPTBP & 0.976 & 0.017 & & $4.74 \mathrm{e}-3$ & 1.63 \\
\hline \multirow[t]{4}{*}{ Dual indicator } & Pd-TFPP & 0.973 & 0.091 & 11.38 & $4.02 \mathrm{e}-1$ & 0.03 \\
\hline & Pt-TFPP & 0.891 & 0.008 & & $8.00 \mathrm{e}-3$ & --- \\
\hline & Pd-TPTBP & 0.976 & 0.052 & 2.00 & $3.16 \mathrm{e}-1$ & 1.00 \\
\hline & Pt-TPTBP & 0.969 & 0.026 & & $4.10 \mathrm{e}-2$ & --- \\
\hline
\end{tabular}

Table S 2 Calibration parameters for the single- and dual-indicator sensors using the simplified Stern-Volmer Fit

To validate the signal deconvolution, the superimposed spectra are compared to the theoretical sum of the individual indicators as shown in Figure $\mathrm{S} 3$ and Figure $\mathrm{S} 4$. While Figure $\mathrm{S} 3$ shows the full spectra including the luminescence of the reference dye at $\mathrm{o} \mathrm{hPa}$ and $200 \mathrm{hPa}$, Figure $\mathrm{S} 4$ focuses on the spectral range of the indicator dyes. 
(A) Spectral deviation Pt-TPTBP+MY

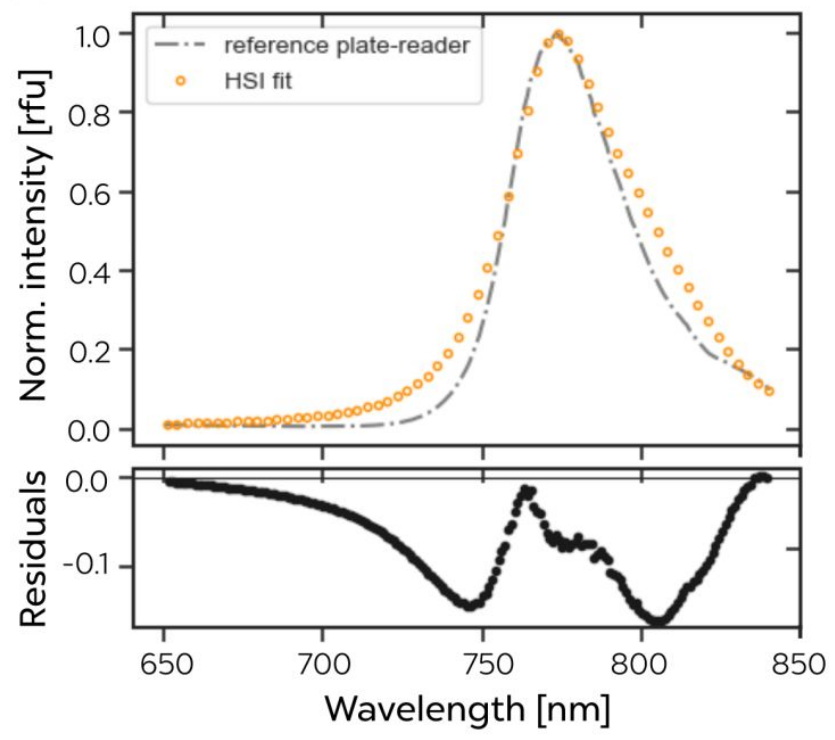

(B) Spectral deviation Pd-TPTBP+MY

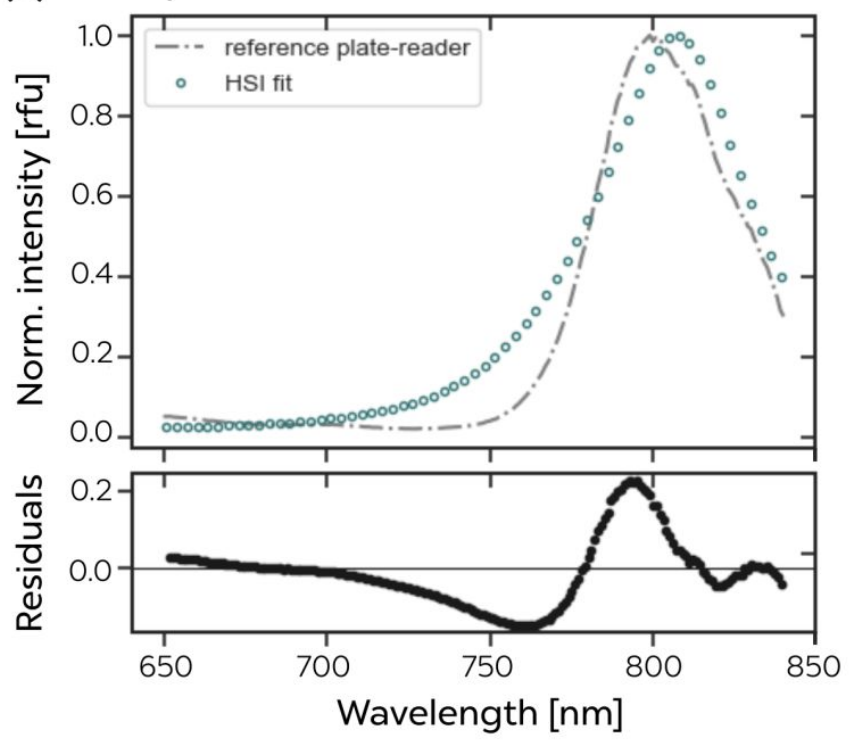

(D) Spectral deviation Pd-TFPP+MY

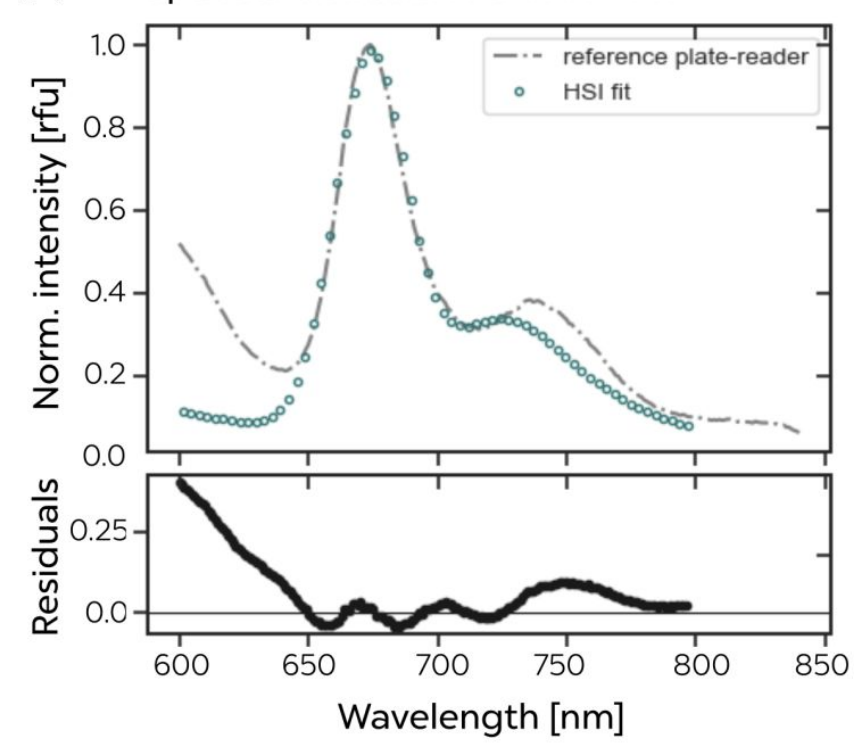

(C) Spectral deviation Pt-TFPP+MY

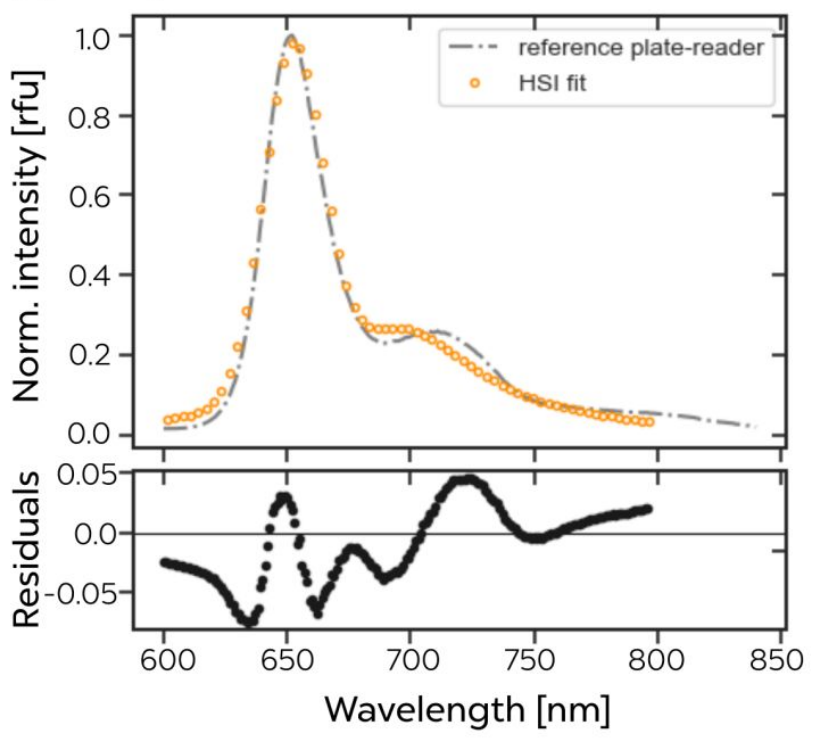

Figure S 2 Spectral deviation of the radiometrically corrected indicator spectra to the corresponding reference spectra recorded on the Clariostar Plus plate-reader. The colored marker dots indicate the distinct measurement point extracted from the hyperspectral cube, while the grey dashed line represents the reference spectrum. In panels (A) and (B) the spectral deviation of the benzoporphyrin-based indicators (Pd-/Pt-TPTBP) is shown, while panels (C) and (D) show the spectral deviation of the porphyrin-based indicators (Pd-/Pt-TFPP). 


\section{Full Spectrum Recorded on the Hyperspectral Camera}

Full fluorescence spectrum of TPTBP dual-sensor

(A) Spectrum at $\mathrm{OhPa}$

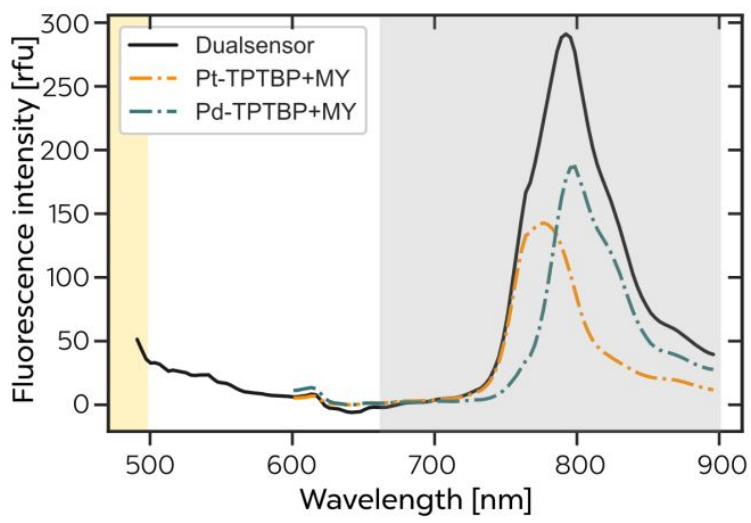

(B) Spectrum at $200 \mathrm{hPa}$

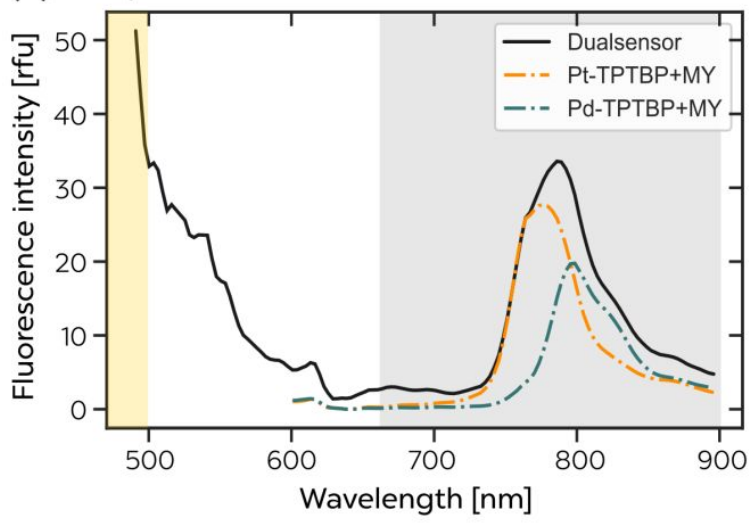

Full fluorescence spectrum of TFPP dual-sensor

(C) Spectrum at $\mathrm{OhPa}$

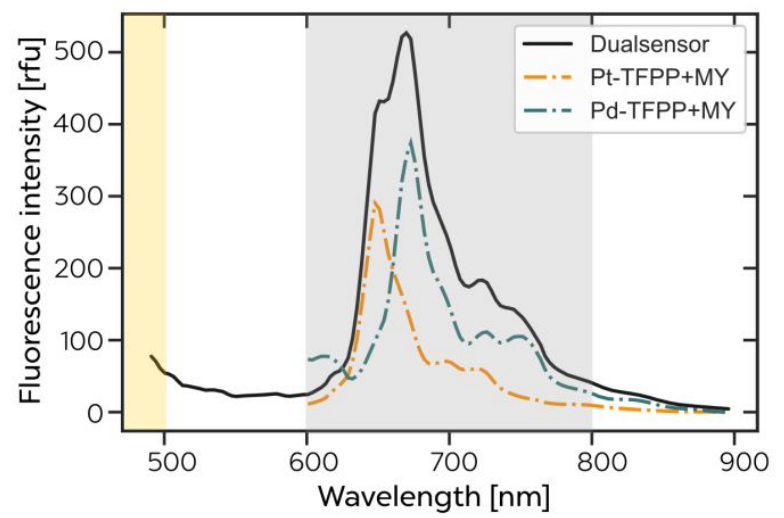

(D) Spectrum at $200 \mathrm{hPa}$

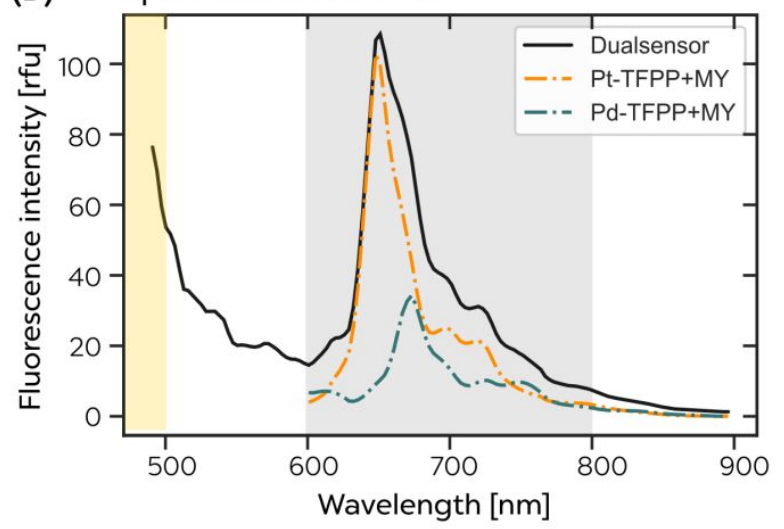

Figure S 3 Full fluorescence spectra recorded on the hyperspectral camera and after radiometric correction. Panels A and B show the benzoporphyrin dual-sensor at $0 \mathrm{hPa}$ and $200 \mathrm{hPa} \mathrm{O}$, respectively, while the porphyrin dual-sensor is shown in panels $\mathrm{C}$ and $\mathrm{D}$. The recorded spectrum of the dual-sensor is marked with a black line (-) and the corresponding spectra of the single indicators are marked as colored dashed lines. The yellow and grey areas indicate the wavelength range in which the integral of the reference dye (yellow area) and the indicator (grey area) was determined. 
Linear Unmixing for all Calibration Points exemplified for the TPTBP Dual-Sensor
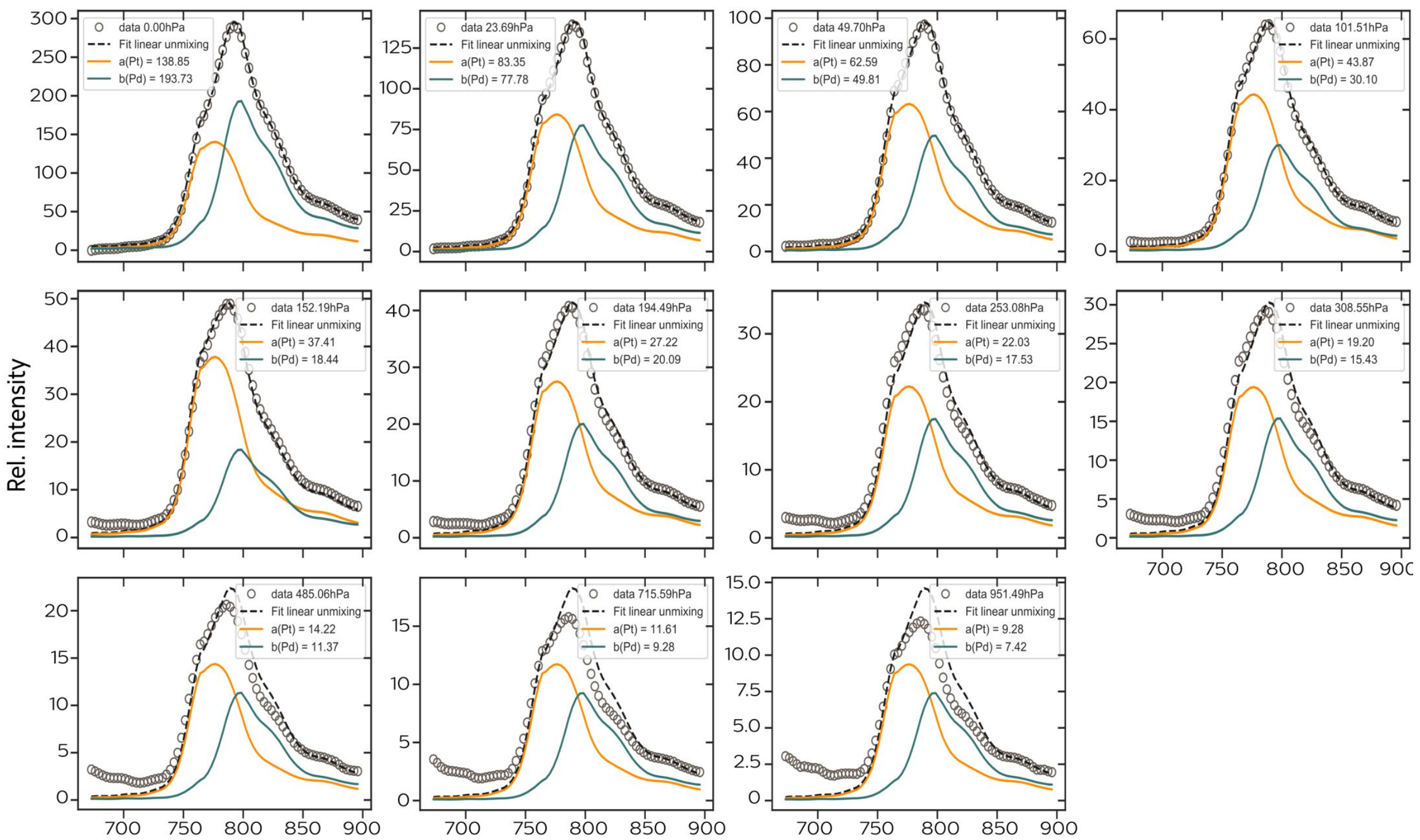

Wavelength $[\mathrm{nm}]$

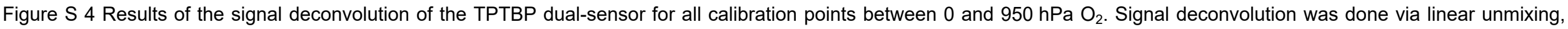

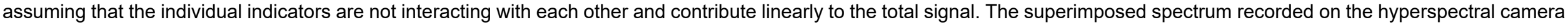

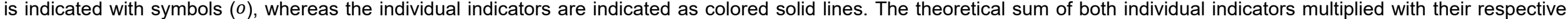

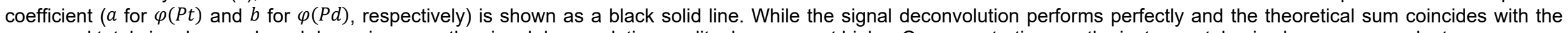
measured total signal over a broad dynamic range, the signal deconvolution quality decreases at higher $\mathrm{O}_{2}$ concentrations as the instrumental noise becomes prevalent. 


\section{Recombination of the Unmixed Dual-Indicators for Joint Imaging}
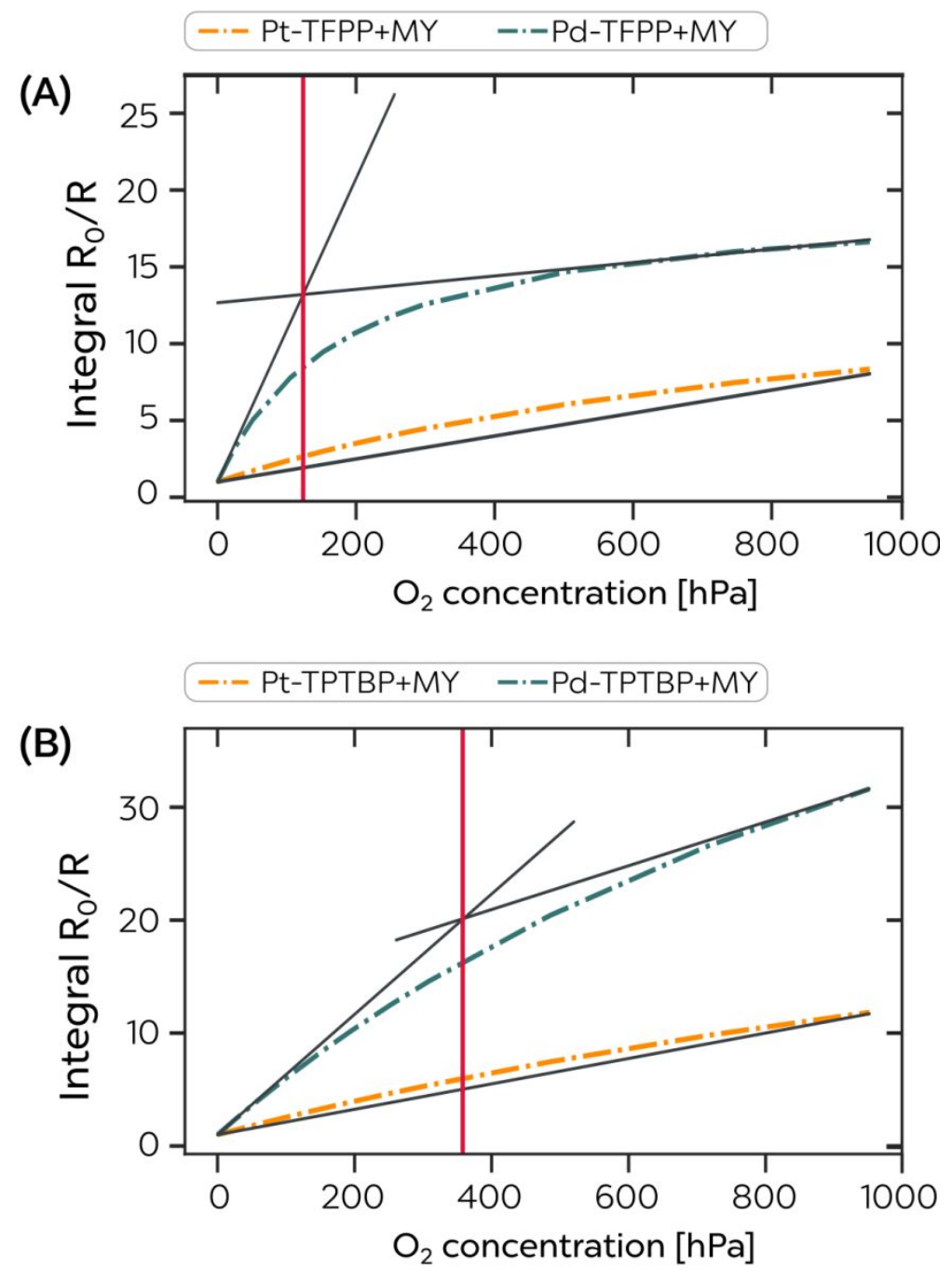

Figure S 5 Comparison of the calibration curves of the respective single $\mathrm{O}_{2}$ indicators in order to recombine the unmixed single indicators for broad-range imaging. Panel A depicts the calibration curves of the TFPP indicators, while panel $B$ shows the calibration curves of the TPTBP indicators. In each case, the calibration curves for the respective single indicators were (partially) linearized and compared in between the indicators to identify the steeper slope. A steeper slope correlates with a higher $\mathrm{O}_{2}$ sensitivity, resulting in a less noisy and more accurate analyte sensing. (A) The Pd-analog (Pd-TFPP) evinces a steeper slope compared to the Pt-analog in the concentration range between 0-125 $\mathrm{hPa} \mathrm{O}$, e.g. Pd-TFPP dominates the dual-indicator sensor within this concentration range, while Pt-TFPP dominates the dual-sensor at higher $\mathrm{O}_{2}$ concentrations. (B) For the benzoporphyrin dual-sensor (Pd-/Pt-TPTBP), the Pd-analog exhibits a steeper slope compared to the respective Pt-analog between 0-355 hPa $\mathrm{O}_{2}$, while the Pt-analog has a steeper slope at higher $\mathrm{O}_{2}$ concentrations. 


\section{Hyperspectral Image and $\mathrm{O}_{2}$ Line Profiles for the TPTBP Dual-Indicator Sensor}

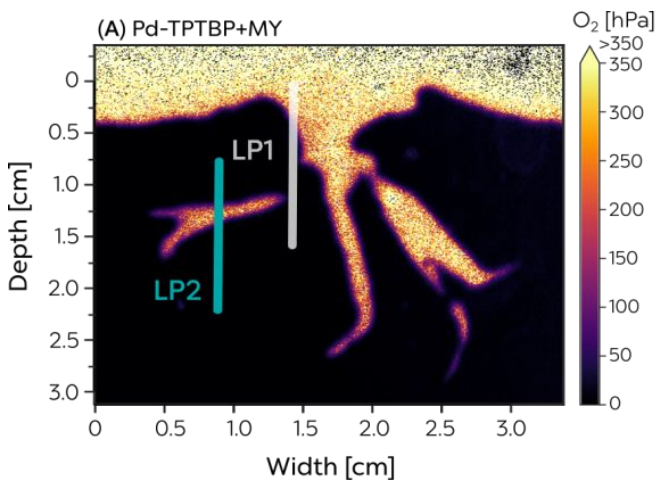

(B) Pt-TPTBP+MY

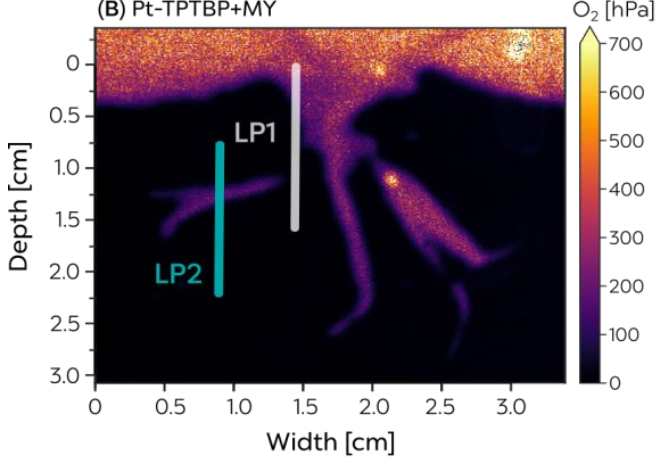

(C) joint sensor information

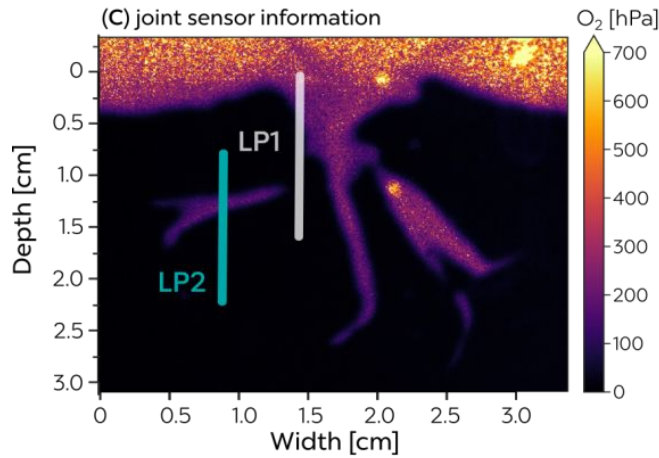

(D) $\mathrm{O}_{2}$ depth profile in LP1

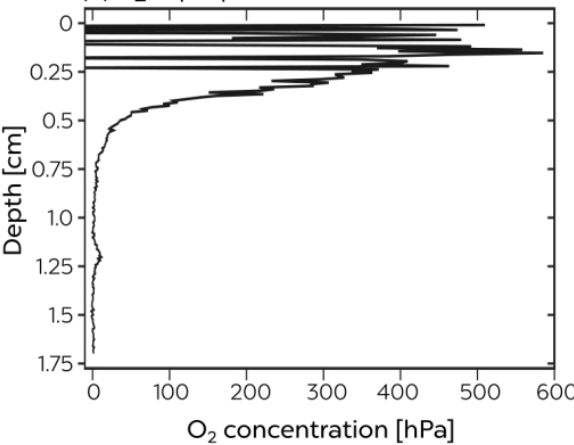

(E) $\mathrm{O}_{2}$ depth profile in LP1

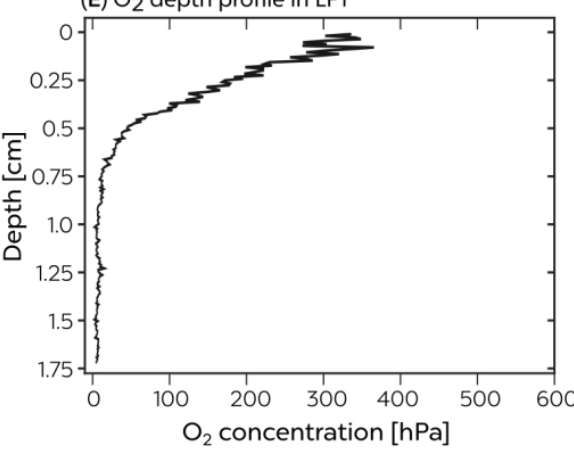

(F) $\mathrm{O}_{2}$ depth profile in LP1

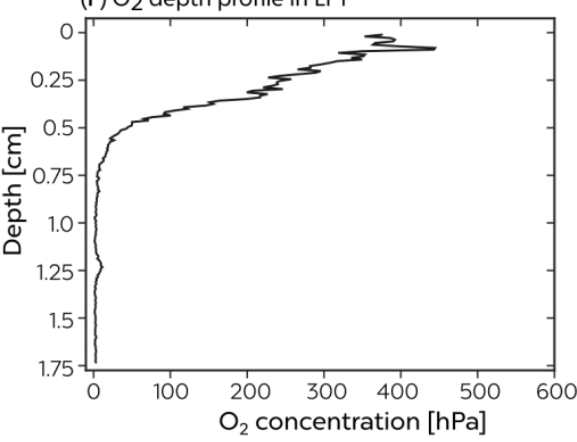

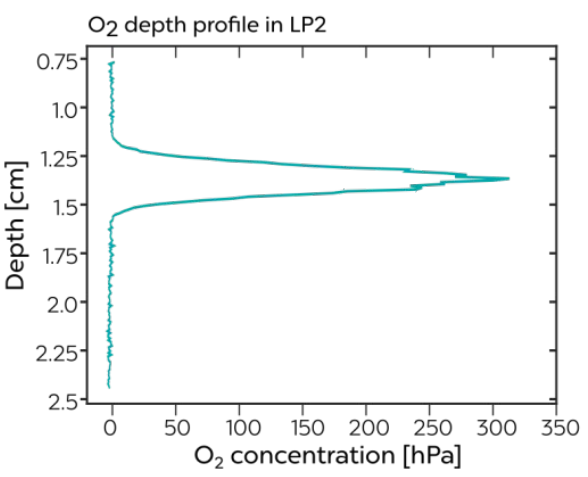
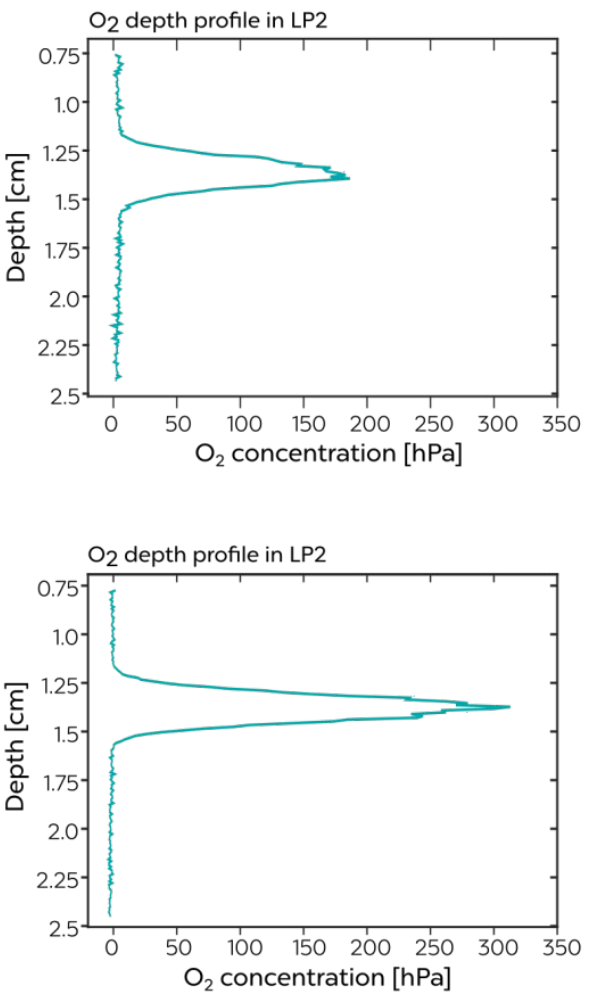

Figure S 6 Mapping of $\mathrm{O}_{2}$ distribution around roots of light-exposed Litorella uniflora using the benzoporphyrin dual-sensor (Pd-/PtTPTBP). Panels A-C on the left visualize the $\mathrm{O}_{2}$ concentration in each pixel of the image frame, which is calculated using the linear unmixing approach. While panel $\mathrm{A}$ and $\mathrm{B}$ show results from the individual indicators upon signal separation, panel $\mathrm{C}$ combines the results of both indicators enabling thus high-resolution broad-range imaging. For $\mathrm{O}_{2}$ concentrations below $355 \mathrm{hPa}$, the dual-indicator sensor relies on information of the Pd-analog, whereas at higher $\mathrm{O}_{2}$ concentrations, the Pt-indicator signal is used. The corresponding panels on the right $(\mathrm{D}-\mathrm{F})$ are examples of oxygen profiles along two line profiles $(\mathrm{LP})$ marked as colored (LP1 = white, LP2 = blue) straight lines in the image frame. 


\section{References}

(1) Shrivastava, A.; Gupta, V. Methods for the Determination of Limit of Detection and Limit of Quantitation of the Analytical Methods. Chronicles Young Sci. 2011, 2 (1), 21. https://doi.org/10.4103/2229-5186.79345.

(2) Pichette, J.; Goossens, T.; Vunckx, K.; Lambrechts, A. Hyperspectral Calibration Method For CMOS-Based Hyperspectral Sensors. In Photonic Instrumentation Engineering IV; Soskind, Y. G., Olson, C., Eds.; SPIE, 2017; Vol. 10110, p 101100H. https://doi.org/10.1117/12.2253617.

(3) Di Roccoa, H. O.; Cruzado, A. The Voigt Profile as a Sum of a Gaussian and a Lorentzian Functions, When the Weight Coefficient Depends Only on the Widths Ratio. Acta Phys. Pol. A 2012, 122 (4), 666-669. https://doi.org/10.12693/aphyspola.122.666.

(4) Horwitz, A. A Version of Simpson's Rule for Multiple Integrals. J. Comput. Appl. Math. 2001, 134 (1-2), 1-11. https://doi.org/10.1016/S0377-0427(00)00444-1.

(5) Carraway, E. R.; Demas, J. N.; DeGraff, B. A.; Bacon, J. R. Photophysics and Photochemistry of Oxygen Sensors Based on Luminescent Transition-Metal Complexes. Anal. Chem. 1991, 63 (4), 337-342. https://doi.org/10.1021/ac00004a007.

(6) Borisov, S. M.; Seifner, R.; Klimant, I. A Novel Planar Optical Sensor for Simultaneous Monitoring of Oxygen, Carbon Dioxide, PH and Temperature. Anal. Bioanal. Chem. 2011, 400 (8), 2463-2474. https://doi.org/10.1007/s00216-0104617-4.

(7) Carraway, E. R.; Demas, J. N.; DeGraff, B. A. Luminescence Quenching Mechanism for Microheterogeneous Systems. Anal. Chem. 1991, 63 (4), 332-336. https://doi.org/10.1021/ac00004a006.

(8) Carraway, E. R.; Demas, J. N.; DeGraff, B. A.; Bacon, J. R. Photophysics and Photochemistry of Oxygen Sensors Based on Luminescent Transition-Metal Complexes. Anal. Chem. 1991, 63 (4), 337-342. https://doi.org/10.1021/ac00004a007.

(9) Miller, J. N.; Miller, J. C. Statistics and Chemometrics for Analytical Chemistry, 5. ed., [N.; Pearson Prentice Hall: Harlow, England, 2009. 